\title{
Publisher Correction: Local sourcing in astronomy
}

Correction to: Nature Astronomy https://doi.org/10.1038/s41550-018-0479-z, published online 1 May 2018.

In the version of this Editorial originally published, we mistakenly wrote that the NAOJ ... may decommission Subaru in favour of other priorities'. In fact, the National Astronomical Observatory of Japan is committed to the long-term operation of the Subaru telescope. In the corrected version that whole sentence has been replaced with: 'It will be critical to maintain such smaller telescopes in the age of the ELTs.'

Published online: 15 May 2018

https://doi.org/10.1038/s41550-018-0496-y 